\title{
DESEMPEÑO ECOFISIOLÓGICO DE CAFÉ (COFFEA ARABICA L.) CV. CASTILLO A LA SOMBRA EN SAN JUAN DE RIOSECO, COLOMBIA
}

\author{
ECOPHYSIOLOGIC PERFORMANCE OF COFFEE \\ (COFFEA ARABICA L.) CV. CASTILLO AT SHADE \\ IN SAN JUAN DE RIOSECO, COLOMBIA
}

${ }^{1}$ Hernán J. Andrade; ${ }^{2}$ Piedad Cecilia Zapata Arango

${ }^{1}$ M. Sc. Agroforestería Tropical, The Nature Conservancy, Bogotá, Colombia. Grupo de Investigación PROECUT, Facultad de Ingeniería Agronómica, Universidad del Tolima;

2 Ph.D. Agroforestería Tropical, Universidad del Tolima, Ibagué, Colombia. The Nature Conservancy. Grupo de Investigación en Producción Agrícola Sostenible- Universidad de Ciencias Aplicadas y Ambientales U.D.C.A.

${ }^{1}$ hjandrade@ut.edu.co; ${ }^{2}$ piedad.zapataltnc.org

\section{Resumen}

La disminución de la radiación disponible, debido a la sombra, modifica las condiciones microclimáticas para el cultivo asociado. Esta modificación es clave para diseñar y manejar sistemas agroforestales. El objetivo de este estudio fue evaluar el efecto de nivel de sombra en el comportamiento ecofisiológico de cafetos (Coffea arabica L.) en San Juan de Rioseco, Cundinamarca, Colombia. Se empleó un diseño experimental aleatorizado con tres tratamientos (sombra baja, media y alta), estimados con fotografías al dosel y Gap Light Analyzer, y cinco repeticiones dadas por los cafetales. En cada cafetal se estableció una parcela de muestreo de $20 \times 50 \mathrm{~m}$, en donde se seleccionaron tres plantas para medir la sombra, la radiación fotosintéticamente activa incidente, la conductancia estomática, la asimilación neta de $\mathrm{CO}_{2}$, la transpiración, el déficit de presión de vapor, la eficiencia en el uso del agua y la concentración de C subestomática con un Sistema de Fotosíntesis Portable - CIRAS 3. La asimilación mayor se registró en cafetos con sombra baja (0-30\%) al medio día, lo que coincidió con la conductancia estomática mayor y el déficit de presión de vapor menor; los valores menores se registraron con alto sombrío (66-75\%) en la tarde. La asimilación de $\mathrm{C}$ se reduce de 44 a 61\%, y la eficiencia en el uso del agua de 41 a $56 \%$ con un incremento de la sombra de bajo a medio y alto, respectivamente. El intervalo óptimo de radiación para los cafetos fue de 42 a $380 \mu \mathrm{mol} \mathrm{m}{ }^{-2}$ $\mathrm{s}^{-1}$, lo cual se encuentra con una sombra entre 20 y $30 \%$ en estas condiciones. Este nivel de sombra es el recomendado en los cafetales en San Juan de Rioseco.

Palabras clave: asimilación de $\mathrm{CO}_{2}$, conductancia estomática, curva de radiación, eficiencia uso de agua, sistemas agroforestales. 


\section{Abstract}

The reduction of available radiation, by shade, modifies microclimatic conditions for associated crops. This modification is a key for the design and management of agroforestry systems. The objective of this study was to evaluate the effect of the shade level on the behaviour of coffee bushes (Coffea arabica L.) in San Juan de Rioseco, Cundinamarca, Colombia. A completely randomized design with three treatments (low, medium and high shade), estimated with photographies to canopy and Gap Light Analyzer, and five replications represented in coffee plantations. It was established a sampling plot of $20 \times 50 \mathrm{~m}$ in each coffee plantation, where three plants were selected to measure shade, photosythetically active radiation, stomatal conductan$\mathrm{ce}$, net assimilation of $\mathrm{CO}_{2}$, transpiration, vapour pressure deficit, water use efficiency and substomatal $\mathrm{CO}_{2}$ concentration with a portable photosynthesis system - CIRAS 3. The greatest net assimilation was recorded in coffee bushes with low shade (0-30\%) at noon, whish coincides with the highest stomatal conductance and the lowest vapour pressure deficit. The lowest values of net assimilation were reached with high shade (66-75\%) in the afternoon. The $\mathrm{CO}_{2}$ assimilation is reduced from 44 to $61 \%$, and the water use efficiency from 41 to $56 \%$ with an increase of shade from low to medium and high levels, respectively. The optimum range for coffee bushes was between 42 and $380 \mu \mathrm{mol} \mathrm{m} \mathrm{m}^{-2} \mathrm{~s}^{-1}$, which is between 20 and $30 \%$ of shade in these conditions. This shade level is the recommended for coffee plantations in San Juan de Rioseco.

Keywords: Assimilation of $\mathrm{CO}_{2}$, stomatal conductance, curve of radiation, water use efficiency, agroforestry systems.

\section{Resumen gráfico}

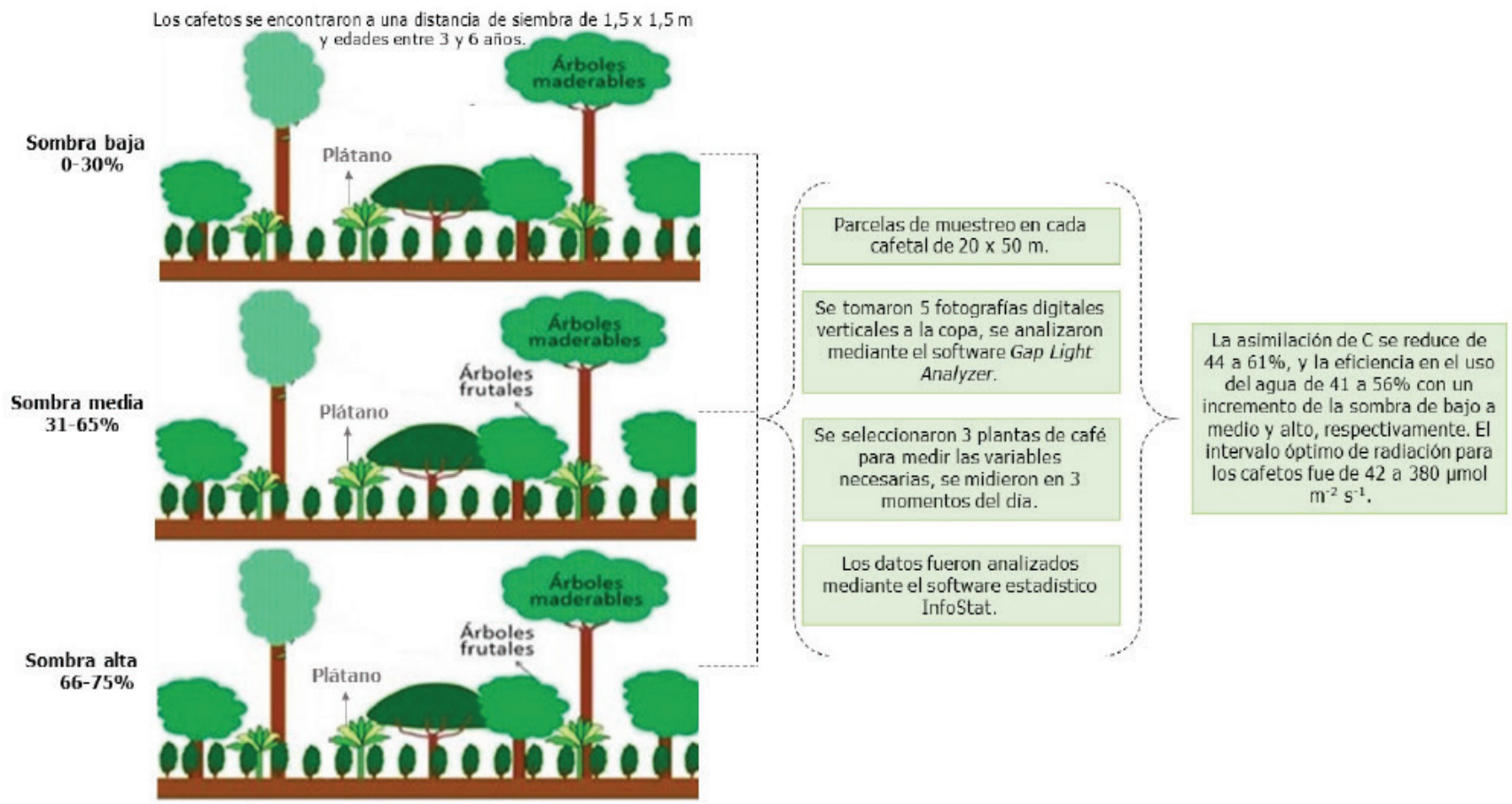




\section{Introducción}

El café (Coffea arabica L.) es uno de los productos más importantes para países en desarrollo de África, Asia y América Latina, por su contribución económica, social y ambiental. Se calcula que la producción mundial de café, entre el 2013 y 2014, fue de 146,8 millones de sacos y América del Sur contribuyó con el $46 \%$. La producción cafetera en Colombia se recuperó después de varios años, con un ascenso del $22 \%$ y una producción de 12,1 millones de sacos (International Coffee Organization, 2016). El café es uno de los productos más importantes de la economía en este país, ya que de él derivan su sustento aproximadamente $500 \mathrm{mil}$ familias y, en el 2015, habían 940.900 hectáreas de este producto sembradas en el 2015, $40 \%$ de ellas con sombrío (Federación Nacional de Cafeteros de Colombia, 2016).

A pesar de la enorme importancia de este sistema de producción, el cambio climático le representa una amenaza, ya que la elevación del $\mathrm{CO}_{2}$ causa un incremento en la temperatura y altera la precipitación, lo cual impactará el comportamiento fisiológico en las zonas de producción (Damatta et al., 2010; Laderach et al., 2010). Los sistemas agroforestales (SAF) juegan un papel importante en la mitigación y adaptación al cambio climático, al combinar los cultivos y especies leñosas perennes, siendo la plasticidad de las plantas fundamental para su adaptación (Calfapietra et al., 2010; Gratiani, 2014).

La fotosíntesis es fuertemente afectada por factores ambientales como la radiación fotosintéticamente activa incidente, la disponibilidad de agua, la temperatura, la humedad relativa y el nivel ambiental de $\mathrm{CO}_{2}$ (Taiz y Zeiger, 2006; Castilla, 2013; Martins et al., 2014). El comportamiento ecofisiológico de las plantas se puede estudiar con las curvas de luz, siendo el punto de compensación aquel donde la incorporación de $\mathrm{CO}_{2}$ en la fotosíntesis es igual a la liberación de $\mathrm{CO}_{2}$ en la respiración; mientras que en el punto de saturación no hay un aumento en la incorporación de $\mathrm{CO}_{2}$ a pesar del incremento de la radiación. El objetivo de este estudio fue evaluar el efecto de nivel de sombra en el comportamiento ecofisiológico de cafetos (Coffea arabica L.) en San Juan de Rioseco, Cundinamarca, Colombia. Los resultados son importantes para el diseño y manejo de SAF con café, principalmente en términos de sombrío y permitirán que los productores logren sistemas ganar-ganar, que permitan incrementar la producción y ofrecer servicios ambientales (Andrade et al., 2014).

\section{Materiales y métodos}

\section{Área de estudio}

El estudio se realizó en el municipio de San Juan de Rioseco, departamento de Cundinamarca, Colombia (450'55" N, 743'14" O, altitud promedio de $1303 \mathrm{~m}$, temperatura media anual de $21^{\circ} \mathrm{C}$, precipitación promedio de $1.313 \mathrm{~mm}$ año-1 y humedad relativa promedio de 68\%). En el municipio ocurren dos estaciones lluviosas, la primera, desde comienzos de abril hasta finales de junio $y$, la segunda, de septiembre a noviembre. Los suelos del área tienen contenido bajo de materia orgánica $(2,3-4,9 \%)$, pendientes pronunciadas (25-50\%), susceptibilidad alta a la erosión, poca profundidad y drenaje y baja capacidad de retención de humedad. La economía del municipio se basa principalmente en la caficultura y la ganadería bovina, ya que las estadísticas del 2013 señalaban la existencia de 13.494 ha de cultivos permanentes, de los cuales el café representaba el 47\% (Corporación Autónoma Regional de Cundinamarca, 2016). 


\section{Unidades de muestreo}

Se evaluaron cinco sistemas de producción de café por cada nivel de sombrío: bajo (SB) de 0 a $30 \%$, medio (SM) de 31 a $65 \%$ y alto (SA) entre 66 y $75 \%$. La sombra en cada cafetal se estimó en una parcela de muestreo de $20 \times 50 \mathrm{~m}$ donde se tomaron cinco fotografías digitales verticales a la copa, empleando un lente plano a una altura de 1,5 m (arriba de los cafetos). Estas fotografías fueron analizadas con el software libre Gap Light Analyzer - GLA (Frazer et al., 1999).

Los lotes seleccionados tenían una distancia de siembra de café $c v$. Castillo de 1,5 51,5 $\mathrm{m}$ y edades entre tres y seis años. Los sitios de muestreo se encontraban a una altitud de entre 1.322 a $1.741 \mathrm{~m}$ con fincas que empleaban fertilización inorgánica y estaban en contextura franco-arcillosa, $\mathrm{pH}$ entre 4,8 y 5,0 y pendientes de 25 a $50 \%$.

Los cafetales con sombrío bajo tuvieron una abundancia de 320 pseudotallos ha-1 de plátano y 56 árboles ha-1 agrupados en 10 especies, siendo Inga edulis, Cordia alliodora y Crescentia cujete las más abundantes. Los árboles tuvieron en promedio un diámetro a la altura del pecho (dap) de $23,5 \mathrm{~cm}$ y altura total de 8,8 $\mathrm{m}$. En cafetales con sombra media, el dosel de sombra estuvo conformado por 166 árboles $\mathrm{ha}^{-1}$ de 15 especies, siendo $I$. edulis (con 62 individuos ha-1) la especie más representativa, seguida de Citrus sinensis, C. alliodora y $C$. cujete y 272 seudotallos de plátano ha-1. Los árboles registraron un dap promedio de 20,7 $\mathrm{cm}$ y una altura total de 9,3 m. Los SAF con sombrío alto tenían una abundancia de 142 árboles ha-1 representados en 11 especies y 706 seudotallos de plátano (Musa AAB) ha-1. En este nivel de sombra, $I$. edulis fue la especie más abundante con 40 individuos ha-1, seguida de C. sinensis, Tabebuia rosea y C. alliodora. Los árboles registraron un dap promedio de $22,9 \mathrm{~cm}$ y una altura total de $9,5 \mathrm{~m}$.

\section{Evaluaciones ecofisiológicas}

En cada parcela de muestreo se seleccionaron tres plantas de café representativas del sistema de producción y en cada planta se escogió una hoja sana por estrato (bajo, medio y alto) del cuarto par a partir del ápice de la rama hacia el tallo. Se seleccionaron ramas con orientación al norte, buscando reducir la variabilidad espacial y temporal. Se monitoreó la radiación incidente (RAFAi) (tomado como el flujo fotosintético de fotones), la conductancia estomática (Gs), la asimilación neta de $\mathrm{CO}_{2}$ $(A)$, la transpiración $(T)$, el déficit de presión de vapor (DPV), la eficiencia en el uso del agua (EUA), la concentración de carbono subestomática ( $\mathrm{Ci}$ ) y la humedad relativa con un Sistema de Fotosíntesis Portable - CIRAS-3 Portable Photosynthesis System (PP Systems Inc., Amesbury, MA, USA) y una cubeta de hoja universal $P C L-3$ que incluye un analizador infrarrojo de gases $\mathrm{CO}_{2} / \mathrm{H}_{2} \mathrm{O}$. Se realizó una medición en diciembre de 2014 en tres momentos del día: entre las 9 y 10 am, 12 m a 1 pm y 3 a 4 pm, luego de estabilizar el equipo al lograr un diferencial de $\mathrm{CO}_{2}$ de $0 \pm 0,5 \mathrm{mb}$.

\section{Análisis estadístico}

Los datos se analizaron con un modelo mixto lineal generalizado - GLMM en InfoStat que utiliza la interfase de la plataforma $\mathrm{R}$, debido a su falta de normalidad en su distribución. El nivel de sombrío, la hora de medición y su interacción se consideraron como efectos fijos; mientras que el sitio y la planta se incluyeron como efectos aleatorios. Se empleó un diseño experimental completamente al azar con tres tratamientos (sombra baja, media y alta), y cinco repeticiones dados por los cafetales.

Con una curva de regresión lineal elaborada con transformaciones logarítmicas que explican la $A$ en función de la RAFAi se estimó el punto de compensación de radiación cuando la A tuvo valor de 0 y el punto de saturación 
lumínica cuando la pendiente de la curva fue 0. La selección del modelo se llevó a cabo empleando los criterios de información de Akaike (AIC) y Bayesiano (BIC) y las pruebas de razón de verosimilitud (LRT). El mejor modelo fue el que presentó los menores valores de AIC y BIC. En los casos en que se rechazó la hipótesis de igualdad de tratamientos, se utilizó la prueba de comparación de medias LSD de Fisher con una significancia de 0,05 y 0,01. Adicionalmente, se estudió la relación entre las variables ecofisiológicas de los cafetos y el nivel de sombrío mediante un análisis de correlación de Pearson. Todos los análisis estadísticos se realizaron en el software InfoStat (Di Rienzo et al., 2016).

\section{Resultados y Discusión}

\section{Relaciones fotosintéticas en cafetos}

Se detectó una interacción significativa en la RAFAi $(p<0,01)$ y en la Gs $(p=0,05)$ entre el nivel de sombrío y la hora de la medición. La mayor RAFAi se presentó en cafetos con SB en la mañana, mientras que el menor valor fue registrado en SA en la mañana (Tabla 1 ). Sin embargo, en términos generales, la RAFAi fue estadísticamente mayor $(p<0,01)$ en la mañana, disminuyendo hacia el mediodía y en la tarde $\left(195,5,144,4\right.$ y $68,3 \mu \mathrm{mol} \mathrm{m} \mathrm{m}^{-2} \mathrm{~s}^{-1}$, respectivamente). La RAFAi fue significativamente superior ( $p=0,01)$ en plantas de café sometidas a SB, que en aquellas que crecían en SM y SA $\left(264,6,85,9\right.$ y $57,6 \mu \mathrm{mol} \mathrm{m} \mathrm{m}^{-2} \mathrm{~s}^{-1}$, respectivamente). La Gs fue superior estadísticamente $(p>0,05)$ independientemente del nivel de sombrío en la mañana y al mediodía que en la tarde: 247,1 vs 235,3 vs $157,9 \mu \mathrm{mol} \mathrm{H}_{2} \mathrm{O} \mathrm{m}^{-2} \mathrm{~s}^{-1}$, respectivamente. La Gs se afectó ligeramente por la sombra ( $p=0,03 ; r=-0,11 ;$ Tabla 2; Figura 1). Por efecto de densidad de leñosas perennes y musáceas, la RAFAi se redujo en este estudio un 68 y $78 \%$ al pasar de niveles de sombra de $22 \%$ a 58 y $72 \%$ respectivamente.

Tabla 1. Comportamiento ecofisiológico de café (Coffea arabica L) cv. Castillo con tres niveles de sombra y en tres momentos del día en SAF en San Juan de Ríoseco, Cundinamarca, Colombia.

\begin{tabular}{|c|c|c|c|c|c|c|c|}
\hline $\begin{array}{c}\text { Hora/ } \\
\text { nivel de } \\
\text { sombra }\end{array}$ & $\begin{array}{c}\text { Radiación } \\
\text { incidente } \\
(\mu \mathrm{mol} \\
\left.\mathrm{m}^{-2} \mathrm{~s}^{-1}\right)\end{array}$ & $\begin{array}{c}\text { Conductancia } \\
\text { estomática } \\
\left(\mu \mathrm{mol} \mathrm{H}_{2} \mathrm{O} \mathrm{m}^{-2}\right. \\
\left.\mathrm{s}^{-1}\right)\end{array}$ & 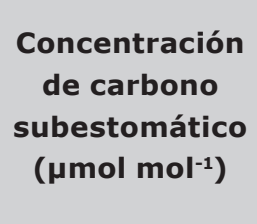 & $\begin{array}{c}\text { Tasa de } \\
\text { asimilación } \\
\text { neta de } \mathrm{CO}_{2} \\
\left(\mu \mathrm{mol} \mathrm{CO}_{2}\right. \\
\left.\mathrm{m}^{-2} \mathrm{~s}^{-1}\right)\end{array}$ & $\begin{array}{c}\text { Tasa de } \\
\text { transpiración } \\
\left(\mu \mathrm{mol} \mathrm{H}_{2} \mathrm{O}\right. \\
\left.\mathrm{m}^{-2} \mathrm{~s}^{-1}\right)\end{array}$ & $\begin{array}{l}\text { Déficit de } \\
\text { presión de } \\
\text { vapor } \\
(\mathrm{kPa})\end{array}$ & $\begin{array}{c}\text { Eficiencia en } \\
\text { el uso del } \\
\text { agua ( } \mu \mathrm{mol}^{-} \\
{ }^{1} \mathrm{CO}_{2} \mathrm{~mol}^{-1} \\
\left.\mathrm{H}_{2} \mathrm{O}\right)\end{array}$ \\
\hline \multicolumn{8}{|l|}{ MAÑANA } \\
\hline Alto & $44,1 \pm 15,3^{f}$ & $242,9 \pm 32,0^{a b c}$ & $340,8 \pm 7,6^{a}$ & $2,9 \pm 0,7^{d}$ & $4,0 \pm 0,3^{\mathrm{abc}}$ & $1,9 \pm 0,2^{\mathrm{ab}}$ & $0,8 \pm 0,2^{\text {ef }}$ \\
\hline Medio & $114,6 \pm 17,9^{c}$ & $247,9 \pm 31,6^{a b c}$ & $319,7 \pm 7,0^{b}$ & $5,6 \pm 0,7^{b c}$ & $4,1 \pm 0,3^{\mathrm{ab}}$ & $1,9 \pm 0,2^{\mathrm{ab}}$ & $1,4 \pm 0,2^{\text {de }}$ \\
\hline Bajo & $427,9 \pm 43,0^{\mathrm{a}}$ & $251,5 \pm 32,2^{b}$ & $268,1 \pm 7,9^{c}$ & $10,8 \pm 0,9^{a}$ & $4,6 \pm 0,3^{a}$ & $2,2 \pm 0,2^{\mathrm{a}}$ & $2,1 \pm 0,2^{b}$ \\
\hline \multicolumn{8}{|c|}{ MEDIO DÍA } \\
\hline Alto & $71,7 \pm 15,6^{\text {cde }}$ & $210,6 \pm 32,0^{\mathrm{abc}}$ & $334,1 \pm 7,6^{\mathrm{ab}}$ & $3,4 \pm 0,7^{d}$ & $3,4 \pm 0,3^{\mathrm{bcd}}$ & $1,7 \pm 0,2^{\mathrm{ab}}$ & $1,1 \pm 0,2^{\text {def }}$ \\
\hline Medio & $92,2 \pm 16,5^{\mathrm{cd}}$ & $222,2 \pm 31,6^{a b c}$ & $316,1 \pm 7,0^{b}$ & $6,6 \pm 0,7^{b}$ & $3,4 \pm 0,3^{\mathrm{cd}}$ & $1,6 \pm 0,2^{b}$ & $1,8 \pm 0,2^{\mathrm{bc}}$ \\
\hline Bajo & $269,3 \pm 23,8^{b}$ & $272,9 \pm 32,2^{a}$ & $278,1 \pm 7,9^{c}$ & $11,7 \pm 0,9^{a}$ & $3,9 \pm 0,3^{b c}$ & $1,5 \pm 0,2^{b}$ & $3,1 \pm 0,2^{\mathrm{a}}$ \\
\hline
\end{tabular}


Continuación tabla 1

\begin{tabular}{|c|c|c|c|c|c|c|c|}
\hline $\begin{array}{c}\text { Hora/ } \\
\text { nivel de } \\
\text { sombra }\end{array}$ & $\begin{array}{c}\text { Radiación } \\
\text { incidente } \\
(\mu \mathrm{mol} \\
\left.\mathrm{m}^{-2} \mathrm{~s}^{-1}\right)\end{array}$ & 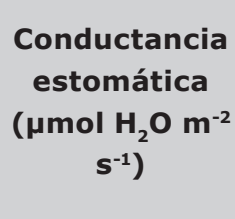 & 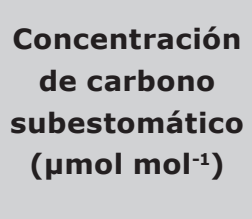 & $\begin{array}{c}\text { Tasa de } \\
\text { asimilación } \\
\text { neta de } \mathrm{CO}_{2} \\
\left(\mu \mathrm{mol} \mathrm{CO}_{2}\right. \\
\left.\mathrm{m}^{-2} \mathrm{~s}^{-1}\right)\end{array}$ & $\begin{array}{c}\text { Tasa de } \\
\text { transpiración } \\
\left(\mu \mathrm{mol} \mathrm{H}_{2} \mathrm{O}\right. \\
\left.\mathrm{m}^{-2} \mathrm{~s}^{-1}\right)\end{array}$ & $\begin{array}{l}\text { Déficit de } \\
\text { presión de } \\
\text { vapor } \\
\text { (kPa) }\end{array}$ & $\begin{array}{c}\text { Eficiencia en } \\
\text { el uso del } \\
\text { agua ( } \mu \mathrm{mol}^{-} \\
{ }^{1} \mathrm{CO}_{2} \mathrm{~mol}^{-1} \\
\left.\mathrm{H}_{2} \mathrm{O}\right)\end{array}$ \\
\hline \multicolumn{8}{|l|}{ TARDE } \\
\hline Alto & $57,1 \pm 14,9^{\text {de }}$ & $122,5 \pm 032,0^{d}$ & $329,6 \pm 7,6^{\mathrm{ab}}$ & $3,3 \pm 0,7^{d}$ & $2,1 \pm 0,3^{e}$ & $2,0 \pm 0,2^{\mathrm{ab}}$ & $1,1 \pm 0,2^{\text {def }}$ \\
\hline Medio & $51,1 \pm 15,4^{\mathrm{ef}}$ & $180,6 \pm 31,6^{\mathrm{bcd}}$ & $341,2 \pm 7,0^{a}$ & $2,3 \pm 0,7^{d}$ & $2,9 \pm 0,3^{\text {de }}$ & $2,0 \pm 0,2^{a}$ & $0,8 \pm 0,2^{f}$ \\
\hline Bajo & $96,6 \pm 16,5^{\mathrm{cd}}$ & $170,7 \pm 32,2^{\mathrm{cd}}$ & $327,2 \pm 7,9^{a b}$ & $3,8 \pm 0,9^{c d}$ & $2,9 \pm 0,3^{\text {de }}$ & $1,8 \pm 0,2^{\mathrm{ab}}$ & $1,5 \pm 0,2^{\mathrm{cd}}$ \\
\hline
\end{tabular}

Valores corresponden a media \pm error estándar. Letras diferentes en cada columna indican diferencias estadísticas $(p<0,05)$.

Tabla 2. Coeficiente de correlación de Pearson entre la sombra y variables ecofisiológicas de cafetos (Coffea arabica L.) cv. Castillo en SAF en San Juan de Ríoseco, Cundinamarca, Colombia.

\begin{tabular}{|c|c|c|c|c|c|c|c|c|c|}
\hline & Sombra & $\begin{array}{l}\text { Radiación } \\
\text { incidente }\end{array}$ & $\begin{array}{c}\text { Conductan- } \\
\text { cia estomá- } \\
\text { tica }\end{array}$ & $\begin{array}{c}\text { Tasa de } \\
\text { asimila- } \\
\text { ción de } \\
\mathrm{CO}_{2}\end{array}$ & $\begin{array}{c}\text { Tasa de } \\
\text { transpira- } \\
\text { ción }\end{array}$ & $\begin{array}{l}\text { Déficit de } \\
\text { presión } \\
\text { de vapor }\end{array}$ & $\begin{array}{l}\text { Eficiencia } \\
\text { de uso } \\
\text { de agua }\end{array}$ & $\begin{array}{l}\text { Concen- } \\
\text { tración de } \\
\text { carbono } \\
\text { subestomá- } \\
\text { tico }\end{array}$ & $\begin{array}{c}\text { Humedad } \\
\text { relativa }\end{array}$ \\
\hline Sombra & & $<0,01$ & 0,03 & $<0,01$ & $<0,01$ & 0,36 & $<0,01$ & $<0,01$ & $<0,01$ \\
\hline $\begin{array}{l}\text { Radiación } \\
\text { incidente }\end{array}$ & $-0,36$ & - & 0,68 & $<0,01$ & $<0,01$ & $<0,01$ & $<0,01$ & $<0,01$ & 0,92 \\
\hline $\begin{array}{l}\text { Conductancia } \\
\text { estomática }\end{array}$ & $-0,11$ & 0,02 & - & $<0,01$ & $<0,01$ & $<0,01$ & $<0,01$ & 0,46 & $<0,01$ \\
\hline $\begin{array}{l}\text { Tasa de } \\
\text { asimilación } \\
\text { de } \mathrm{CO}_{2}\end{array}$ & $-0,44$ & 0,46 & 0,57 & - & $<0,01$ & $<0,01$ & $<0,01$ & $<0,01$ & $<0,01$ \\
\hline $\begin{array}{l}\text { Tasa de } \\
\text { transpiración }\end{array}$ & $-0,22$ & 0,34 & 0,78 & 0,60 & - & 0,01 & $<0,01$ & $<0,01$ & $<0,01$ \\
\hline $\begin{array}{l}\text { Déficit de } \\
\text { presión de } \\
\text { vapor }\end{array}$ & $-0,05$ & 0,48 & $-0,60$ & $-0,19$ & $-0,12$ & - & $<0,01$ & $<0,01$ & $<0,01$ \\
\hline $\begin{array}{l}\text { Eficiencia en } \\
\text { el uso del } \\
\text { agua }\end{array}$ & $-0,42$ & 0,34 & 0,25 & 0,83 & 0,17 & $-0,20$ & - & $<0,01$ & $<0,01$ \\
\hline $\begin{array}{l}\text { Concentración } \\
\text { de carbono } \\
\text { subestomático }\end{array}$ & 0,42 & $-0,62$ & $-0,04$ & $-0,75$ & $-0,23$ & $-0,28$ & $-0,85$ & - & 0,17 \\
\hline $\begin{array}{l}\text { Humedad } \\
\text { relativa }\end{array}$ & $-0,20$ & 0,01 & 0,72 & 0,50 & 0,38 & $-0,82$ & 0,45 & $-0,07$ & - \\
\hline
\end{tabular}

El triángulo inferior corresponde a los coeficientes de correlación de

Pearson y el triángulo superior se refiere a su nivel de significancia. 

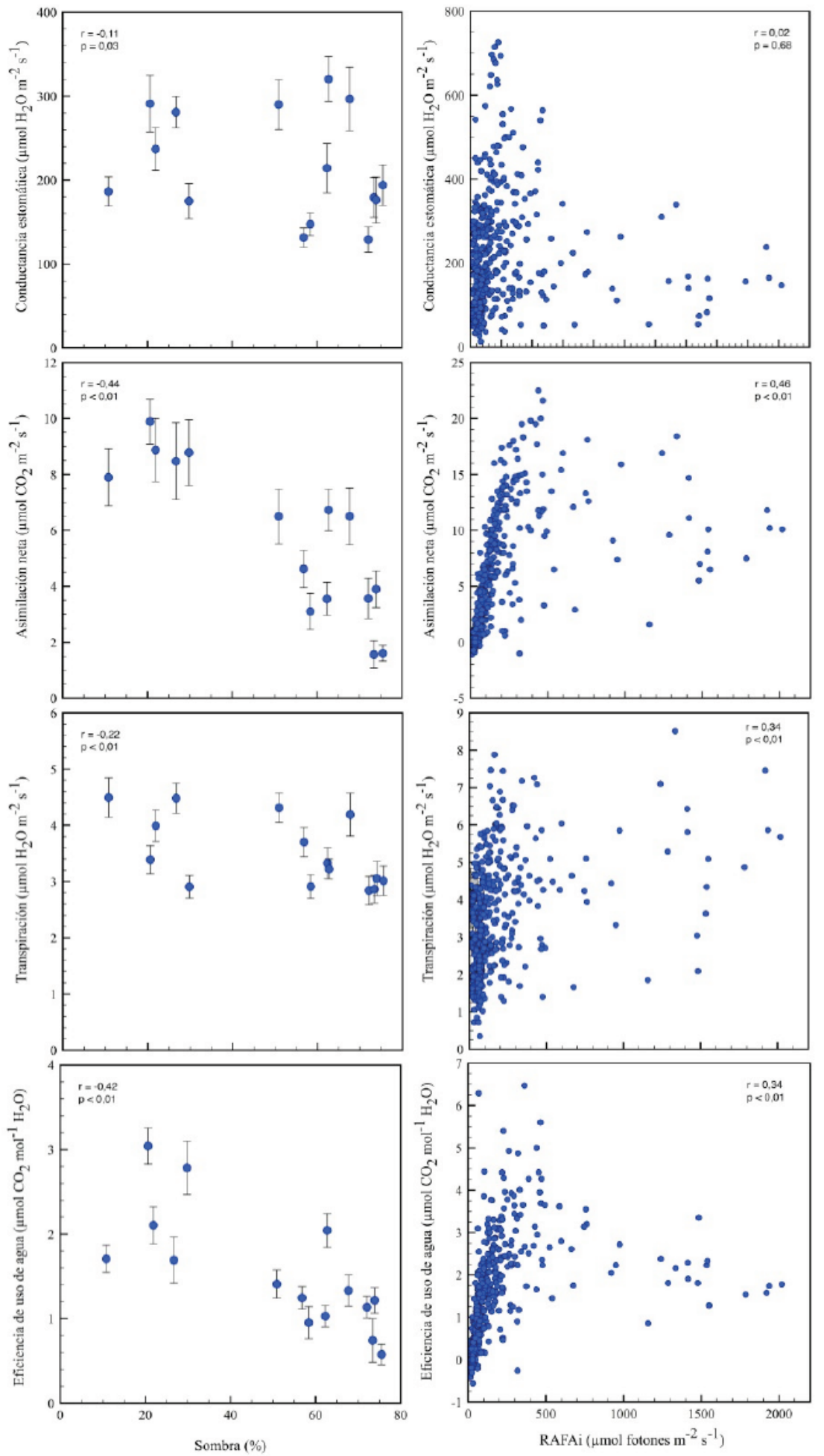

Figura 1. Relación entre el porcentaje de sombra y la radiación fotosintéticamente activa incidente (RAFAi) (dado como el flujo fotosintético de fotones) y variables ecofisiológicas de cafeto (Coffea arabica L.) cv. Castillo en SAF en San Juan de Rioseco, Cundinamarca, Colombia. Las barras de error corresponden a error estándar de la media.

Fuente: Autores. 
En San Juan de Rioseco, la densidad de plátano fue determinante en la sombra y RAFAi para los cafetos, lo que se convierte en un factor clave para el diseño y manejo de Ios SAF con café. La relevancia de la sombra generada por este tipo de plantas radica en que son sombras "densas o lentas" ya que, debido a la baja altura de la base de la copa, la sombra cubre poca área de suelo y se "mueve" lentamente a lo largo del día (Somarriba, 2004). La RAFAi varió a lo largo del día probablemente por cambios en la nubosidad, la orientación del cafetal y del terreno y la inclinación (declinación y azimut) de los rayos solares sobre la superficie. La mayor Gs se registró en las primeras horas del día y disminuyó progresivamente a lo largo del día, coincidiendo con otras investigaciones (López, 2004; Chaves et al., 2012; Rodríguez et al., 2012). Estos mismos autores también encontraron una correlación inversa entre Gs y DVP.
La A fue estadísticamente superior $(p<0,01)$ al medio día y en la mañana en $\mathrm{SB}$, que en la tarde en SA y SM (Tabla 1). El sombrío, independiente del momento del día, presentó diferencias significativas $(p<0,01)$ en la $A$, donde cafetos en SAF con SA y SM fueron estadísticamente iguales ( $p$ $>0,05)$ pero menores a lo registrado en SB: 3,2 y 4,8 vs $8,8 \mu \mathrm{mol} \mathrm{CO}_{2} \mathrm{~m}^{-2} \mathrm{~s}^{-1}$, respectivamente. Se encontró una relación cuadrática entre la sombra y la A (Tabla 2; Figuras 1 y 2); mientras que se presentó una correlación directa y significativa entre Gs y $A$ ( $p<0,01 ; r=0,57$; Tabla 2). En los días de medición, la temperatura ambiental no fue un determinante de la $A$, ya que se presentaron promedios de $22,3{ }^{\circ} \mathrm{C}$, siendo la temperatura elevada (de 30 a $35^{\circ} \mathrm{C}$ ) la que provoca el cierre de estomas (Taiz y Zeiger, 2006). En términos generales, se estimó que la $A$ en cafetales sin sombra llegaría a $8,6 \mu \mathrm{mol} \mathrm{CO}_{2} \mathrm{~m}^{-2} \mathrm{~s}^{-1}$; mientras que la tasa máxima A se alcanzaría con cerca de un $20 \%$ de sombra, hasta llegar a mínimos valores de A con sombras muy altas (> 70\%) (Figura 2).

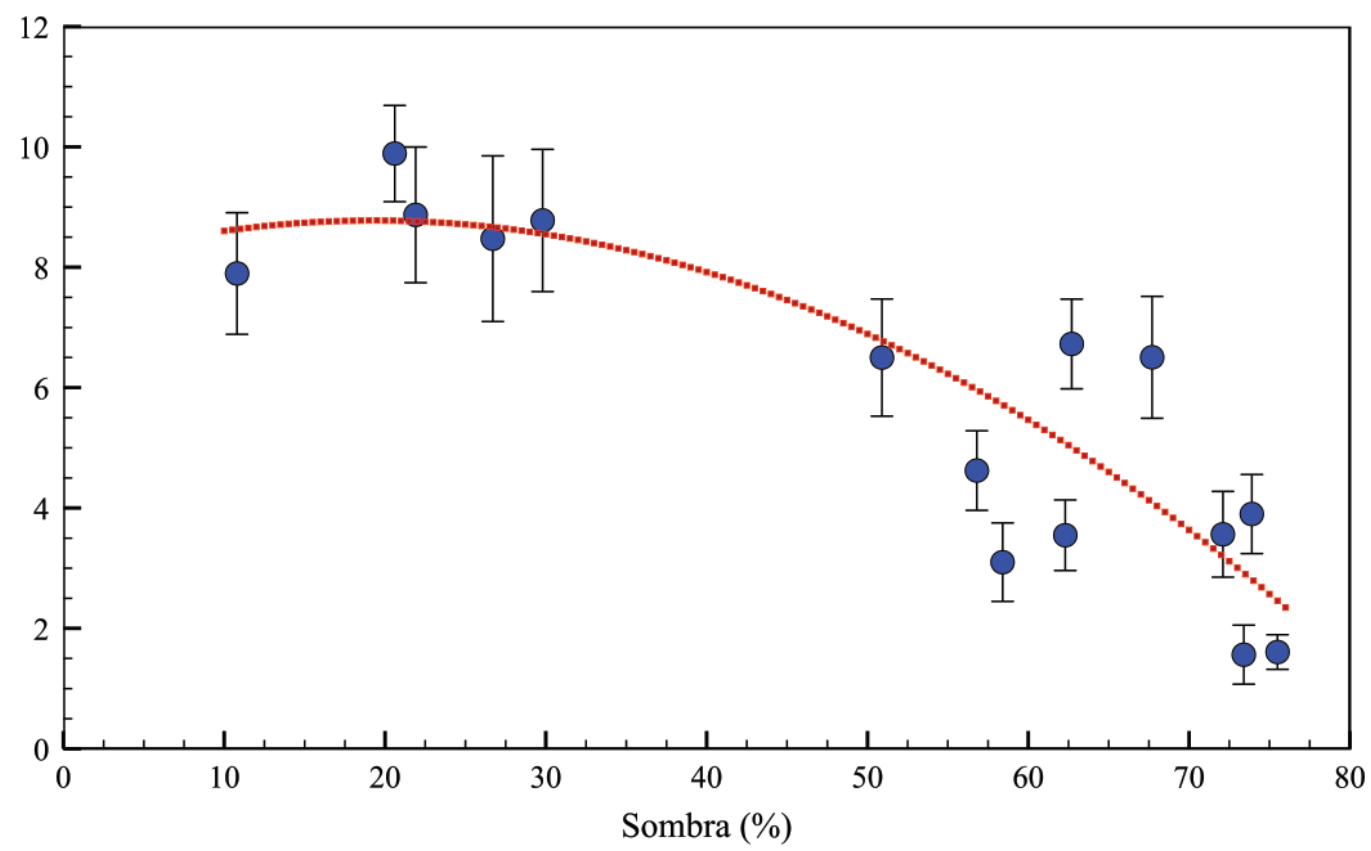

Figura 2. Relación entre la sombra en la asimilación neta de $\mathrm{CO}_{2}(\mathrm{~A})$ de cafetos (Coffea arabica L.) cv. Castillo en sistemas agroforestales en San Juan de Ríoseco, Cundinamarca, Colombia.

Fuente: Autores. 
Aunque se encontró un coeficiente de determinación bajo $\left(R^{2}=0,57\right)$, se observa la tendencia de la $A$ en función de la RAFAi. El punto de compensación de radiación para los cafetos se estimó en $42 \mu \mathrm{mol} \mathrm{m}{ }^{-2} \mathrm{~s}^{-1}$; mientras que el punto de saturación de esta variable microclimática se alcanzó a los $380 \mu \mathrm{mol} \mathrm{m}^{-2}$ $\mathrm{s}^{-1}$ (Figura 3); SAF que suministren RAFAi inferior a este rango se reduce la productividad primaria neta de los cafetos, ya que arrojaría valores negativos, lo que implica una pérdida de asimilados producto de la fotosíntesis.

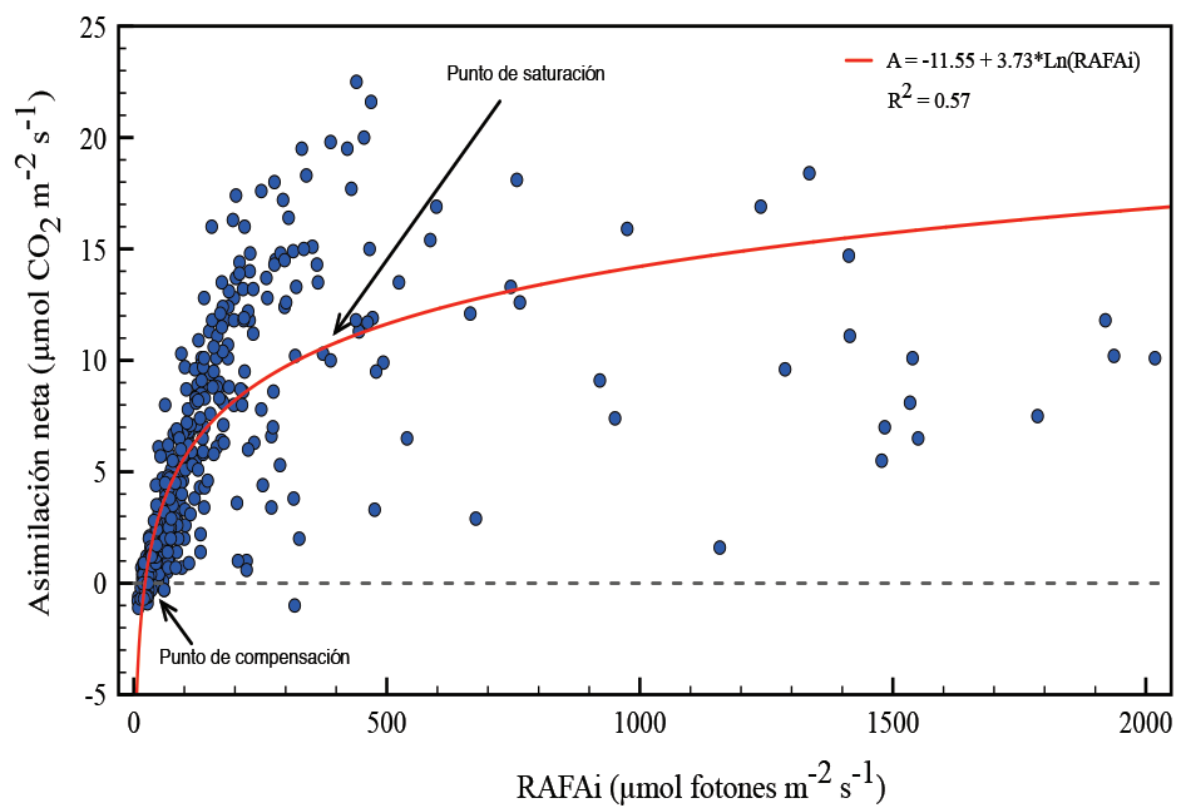

Figura 3. Curva de radiación (relación entre asimilación neta de $\mathrm{CO}_{2}-\mathrm{A}-\mathrm{y}$ radiación fotosintéticamente activa incidente -RAFAi) en cafetos (Coffea arabica L.) cv. Castillo en SAF en San Juan de Ríoseco, Cundinamarca, Colombia.

Fuente: Autores.

Se encontró una interacción estadística ( $p<$ $0,01)$ para la Ci entre el nivel de sombrío y la hora del día, siendo esta variable mayor en SAF con SM en la tarde y SA en la mañana y menor en SB al medio día y en la mañana (Tabla 1). Independiente del nivel de sombrío, se presentaron diferencias significativas $(p<0,01)$ en la Ci entre horas del día, encontrándose los mayores valores en la tarde comparado a la mañana y medio día (332,6 vs 309,5 y 309,4 $\mathrm{mmol} \mathrm{mol}^{-}$ 1 , respectivamente). El nivel de sombrío afectó estadísticamente $(p<0,01)$ la $\mathrm{Ci}$, siendo mayor en SA que en SM y SB (334,8 vs 325,7 y 291,1 $\mu \mathrm{mol} \mathrm{mol}{ }^{-1}$, respectivamente).
Los altos valores de Gs y A en SB al medio día y en la mañana están relacionadas con bajas $\mathrm{Ci}$ en esta investigación, esto coincide con lo propuesto por Taiz y Zeiger (2006) quienes afirman que las bajas concentraciones de $\mathrm{CO}_{2}$ en las hojas ocasionan apertura estomática para satisfacer sus necesidades de insumos para fotosíntesis. Las menores tasas de A encontradas en SAF con sombras altas y medias se deberían a los niveles muy bajos de RAFAi, esto indica que esta variable es probablemente la interacción biofísica más importante en SAF (Andrade et al., 2014). 
En C. arabica cv. Caturra cultivada en el Valle de Orosí en Costa Rica, Franck y Vaast (2009) encontraron que la asimilación de $\mathrm{CO}_{2}$ se limitó fuertemente al pasar de 5,1 $\mu \mathrm{mol} \mathrm{CO}_{2} \mathrm{~m}^{2} \mathrm{~s}^{-1}$ en sistemas a pleno sol a 2,7 $\mu \mathrm{mol} \mathrm{CO}_{2} \mathrm{~m}^{2} \mathrm{~s}^{-1}$ en aquellos con $19 \%$ de RAFAi; mientras que Morais et al. (2012) registraton tasas de asimilación de 5,9 a $8,8 \mu \mathrm{mol} \mathrm{CO}_{2} \mathrm{~m}^{-2} \mathrm{~s}^{-1}$ en café robusta ( $C$. canephora) en Brasil, estos últimos iguales a los presentados en SAF de SB en esta investigación. En contraste, estudios en C. arabica L. Cv Catucaí y plantas de C. arabica cv. Colombia a libre exposición arrojaron tasas de asimilación menores a 5,0 y de 4,1 a $5,0 \mu \mathrm{mol} \mathrm{CO} \mathrm{m}^{-2} \mathrm{~s}^{-1}$, respectivamente (Araujo et al., 2008; Baliza et al., 2012), las cuales resultaron similares a lo registrado en SAF con SM y SA.

Los puntos de compensación y saturación de radiación estimados en el presente estudio contrastaron con los reportados por Rodríguez et al. (2012) para C. canephora (10,7 - 27,6 y 552 - $660 \mu \mathrm{mol} \mathrm{m} \mathrm{m}^{-2} \mathrm{~s}^{-1}$, respectivamente). Sin embargo, este comportamiento fisiológico depende de la especie y de la misma RAFAi, ya que el punto de compensación de luz es mayor en las hojas expuestas al sol y plantas heliófilas que en las láminas foliares sombreadas y plantas umbrófilas (Taiz y Zeiger, 2006; Matos et al., 2009). Este estudio encontró puntos de saturación de 100 a $200 \mu \mathrm{mol} \mathrm{m} \mathrm{m}^{-2} \mathrm{~s}^{-1}$ en cafetos en SAF con sombra media y alta, lo que coincide con Taiz y Zeiger (2006), quienes afirman que una planta umbrófila emplea hasta $150 \mu \mathrm{mol} \mathrm{m} \mathrm{m}^{-2} \mathrm{~s}^{-1}$ para la fotosíntesis y valores RAFAi superiores la podrían saturar, haciendo que los excedentes de energía luminosa deban ser disipados en forma de calor.

\section{Comportamiento hídrico foliar en cafetos}

Se presentó una interacción estadística ( $p=$ 0,04 ) en la $T$ entre el nivel de sombrío y la hora del día, siendo mayor en plantas de SB en la mañana y menor en aquellas de SA durante la tarde (Tabla 1). Sin importar el sombrío, en las horas del día se detectaron diferencias significativas $(p<0,01)$ en la $T$, siendo mayor en las mañanas, seguido del medio día y de la tarde (4,2 vs 3,6 vs $2,7 \mu \mathrm{mol} \mathrm{H}_{2} \mathrm{O} \mathrm{m} \mathrm{m}^{-2} \mathrm{~s}^{-1}$, respectivamente). En contraste, el nivel de sombrío no afectó estadísticamente $(\mathrm{p}=0,27)$ la $\mathrm{T}$; $\sin$ embargo, esta variable ecofisiológica sí se relacionó con la Gs y la $A(p<0,01$ y $r=0,78$; $p$ $<0,01$ y $r=0,60$; Figura 1$)$.

El DVP y la EUA presentaron una interacción estadística $(p<0,01)$ entre el nivel de sombrío y la hora del día, ya que los cafetos en SAF con SB y SM alcanzaron los mayores valores en la mañana y en la tarde, reduciéndose drásticamente hasta alcanzar el mínimo al medio día en SM y SB (Tabla 1). Tal como se esperaba, el DVP en la mañana y tarde fueron significativamente $(p<0,01)$ mayores que al medio día (2,0 vs 1,6 kPa), pero esta variable no fue afectada por el nivel de sombrío ( $p=0,99$ ). Los cafetos que crecían en SAF con SB presentaron la mayor EUA al medio día, variable que fue reduciéndose hasta los mínimos obtenidos en SM en la tarde (Tabla 1).

Contrastando con la T, la EUA sí fue afectada estadísticamente $(p<0,01)$ por el nivel de sombrío, ya que los cafetos que crecían en SAF con SB fueron más eficientes en el uso del agua que aquellos bajo SM y SA $(2,3$ vs 1,3 y $1,0 \mu \mathrm{mol} \mathrm{CO}$ mol$^{-1} \mathrm{H}_{2} \mathrm{O}$, respectivamente). De la misma forma, la EUA fue estadísticamente mayor $(p<0,01)$ a medio día que la mañana y la tarde $\left(2,0\right.$ vs 1,5 vs $1,2 \mu \mathrm{mol} \mathrm{CO}_{2}$ $\mathrm{mol}^{-1} \mathrm{H}_{2} \mathrm{O}$, respectivamente). En este estudio, la tasa transpiracional en cafetos se redujo 9 - $17 \%$ al incrementarse la sombra, lo que es posible que sea causado por una menor Gs, más que a diferencias en el DPV (1,9 vs 1,8 $\mathrm{kPa}$, respectivamente). Baliza et al. (2012) encontraron una menor transpiración en $C$. 
arabica cv Catucaí en sombras altas (65 y $90 \%$ ). La mayor humedad del aire en SAF con SB con respecto a SA y SM $(66,3$ vs 61,5 y $62,7 \%$, respectivamente) podría influir en la apertura de los estomas y por ende, estimular la transpiración (Taiz y Zeiger, 2006). Sin embargo, el ahorro de agua en el presente estudio fue menor que en Chiapas, México, donde un sombrío mayor a $30 \%$ redujo en $32 \%$ la transpiración respecto a sistemas con poca sombra (Lin, 2010). La baja EUA de cafetos en media y alta sombra es un efecto esperado debido al bajo flujo fotosintético de fotones para la fase lumínica de la fotosíntesis, lo que reduce la asimilación neta de $\mathrm{CO}_{2}$ (Taiz y Zeiger, 2006). Al respecto, Cavatte et al. (2012) mencionan que plantas cultivadas a pleno sol muestran una mejor EUA a largo plazo, especialmente en condiciones de sequía.

\section{Conclusiones}

Bajo las condiciones en que se realizó esta investigación, una sombra superior al 30\% afectó negativamente, y de manera drástica, la eficiencia en el uso del agua y las tasas fotosintéticas de los cafetos por la reducción de la radiación disponible, la cual incide en el desempeño del aparato fotosintético. La máxima tasa de asimilación neta en estos cafetos se logró a una sombra de cerca del 20\%.

Las plantas de café cv. Castillo presentan mayor adaptación a sombras de hasta $30 \%$, ya que presentaron tasas de asimilación de $\mathrm{CO}_{2}$ similares a otros cultivares en Colombia y Brasil a plena exposición solar.

Los cafetos en SAF en las condiciones del municipio de San Juan de Rioseco pueden tolerar niveles de radiación fotosintéticamente activa incidente de entre 42 y $380 \mu \mathrm{mol} \mathrm{m} \mathrm{m}^{-2} \mathrm{~s}^{-1}$. En este rango de radiación, los cafetos presentan una productividad primaria neta positiva. Valores mayores al límite superior implican una reducción del componente arbóreo y no mejoran considerablemente sus tasas de asimilación neta de los cafetos. La sombra ideal en este caso oscila entre 20 y $30 \%$.

\section{Agradecimientos}

Esta investigación fue financiada por el Departamento de Cundinamarca por el Sistema General de Regalías - Fondo de Ciencia, Tecnología e Innovación, mediante el Convenio 009, 2014, entre el Departamento de Cundinamarca y la Universidad de Ciencias Aplicadas y Ambientales U.D.C.A, con la participación de la Corporación Autónoma Regional de Cundinamarca y la Corporación Latinoamericana Misión Rural.

A los 15 caficultores donde se realizó la investigación por el acceso a sus fincas, a Héctor Daniel Sandoval y William Cárdenas por su colaboración en la toma de datos. Se agradece la financiación del proyecto con recursos del departamento de Cundinamarca del Sistema General de Regalías - Fondo de Ciencia, Tecnología e Innovación, a través del Convenio 009 de 2014, suscrito entre el departamento de Cundinamarca y la Universidad de Ciencias Aplicadas y Ambientales U.D.C.A, donde participaron, como aliados, la Corporación Autónoma Regional de Cundinamarca y la Corporación Latinoamericana Misión Rural.

\section{Literatura citada}

Andrade, H.J., Marín, L.M., y Pachón, D.P. (2014). Fijación de carbono y porcentaje de sombra en sistemas de producción de café (Coffea arabica L.) en el Líbano, Tolima, Colombia. Bioagro, 26 (2), pp. 127-132.

Araujo, W.L., Dias, P.C., Moraes, G.A., Celin, E.F., Cunha, R.L., Barros, R.S., y Damatta, F.M. (2008). Limitations to photosynthesis in coffee leaves from different canopy positions. Plant Physiology and Biochemistry, 46 (10), pp. 884-890. 
Baliza, D.P., Cunha, R.L., Guimarães, R.J., Barbosa, J.P., Ávila, F.W., and Passos, A.M. (2012). Physiological characteristics and development of coffee plants under different shading levels. Revista Brasileira de Ciencias Agrarias, 7 (1), pp. 37-43.

Calfapietra, C., Gielen, B., Karnosky, D., Ceulemans, R., y Scarascia, G. (2010). Response and potential of agroforestry crops under global change. Environmental Pollution, 158 (4), pp. 1095-1104.

Castilla, N. (2013). Greenhouse technology and management. 2nd Edition. CABI Publishing. Boston, p. 360.

Cavatte, P.C., Oliveira, A.A., Morais, L.E., Martins, S.C., Sanglard, L.M., y Damatta, F.M. (2012). Could shading reduce the negative impacts of drought on coffee? A morphophysiological analysis. Physiologia Plantarum, 144 (2), pp. 111-122.

Chaves, A., Martins, S., Batista, K.D., Celin, E.F., y Damatta, F.M. (2012). Varying leaf-to-fruit ratios affect branch growth and dieback, with little to no effect on photosynthesis, carbohydrate or mineral pools, in different canopy positions of field-grown coffee trees. Environmental and Experimental Botany, 77, pp. 207-218.

Corporación Autónoma Regional de Cundinamarca. (2016). Formulación del plan de ordenación y manejo de la cuenca del Río Magdalena (vertiente oriental del departamento de Cundinamarca). Recuperado de: https://www.car.gov.co/ uploads/files/5ac6789272df3.pdf (Consulta: noviembre de 2017).

Damatta, F.M., Grandis, A., Arenque, B.C., y Buckeridge, M.S. (2010). Impacts of climate changes on crop physiology and food quality. Food Research International, 43 (7), pp. 1814-1823.

Di Rienzo, J.A., Casanoves, F., Balzarini, M.G., Gonzalez, L., Tablada, E.M., y Robledo, C.W. (2016). InfoStat, versión 2016. Grupo InfoStat, FCA, Universidad Nacional de Córdoba. Argentina.

Federación Nacional de Cafeteros de Colombia. (2016). Área cultivada con café según exposición solar por departamento. Recuperado de: http//:www.federaciondecafeteros.org/particulares/es/quienes_somos/119_estadisticas_historicas. (Consulta: diciembre de 2016).

Franck, N., y Vaast, P. (2009). Limitation of coffee leaf photosynthesis by stomatal conductance and light availability under different shade levels. Trees, 23 (4), 761-769.
Frazer, G.W., Canham, C.D., y Lertzman, K.P. (1999). Gap Light Analyzer (GLA), Version 2.0: Imaging software to extract canopy structure and gap light transmission indices from true-colour fisheye photographs, user's manual and program documentation. Simon Fraser University, Burnaby, British Columbia, and the Institute of Ecosystem Studies, Millbrook, New York, p. 40.

Gratiani, L. (2014). Plant phenotypic plasticity in response to environmental factors. Advances in Botany, 2014, 1-17.

International Coffee Organization (2016). Anuario 2013-2014. Fortalecimiento del sector cafetero mundial mediante la cooperación internacional. Recuperado de: http://www.ico.org/documents/ cy2014-15/annual-review-2013-14-electronic-c. pdf. (Consulta: diciembre de 2016).

Laderach, P., Lundy, M., Jarvis, A., Ramirez. J., Perez, E., Schepp, K., y Eitzinger, A. (2010). Predicted impact of climate change on coffee-supply chains. En: Leal Filho W, (Org.). The Economic, Social and Political Elements of Climate Change, pp. 703-723.

Lin, B.B. (2010). The role of agroforestry in reducing water loss through soil evaporation and crop transpiration in coffee agroecosystems. Agricultural and Forest Meteorology, 150 (4), 510-518.

López, J.C. (2004). Comportamiento del intercambio gaseoso de Coffea arabica $L$. en tres altitudes de la zona cafetera central colombiana. Cenicafé, 55 (3), pp. 202-212.

Martins, S.C., Galmés, J., Cavatte, P.C., Pereira, L.F., Ventrella, M.C., y Damatta, F.M. (2014). Understanding the low photosynthetic rates of sun and shade coffee leaves: bridging the gap on the relative roles of hydraulic, diffusive and biochemical constraints to photosynthesis. Plos One, 9 (4), pp. 1-10.

Matos, F.S., Wolfgramm, R., Gonçalves, F.V., Cavatte, P.C., Ventrella, M.C., y Damatta, F.M. (2009). Phenotypic plasticity in response to light in the coffee tree. Environmental and Experimental Botany, 67 (2), pp. 421-427.

Morais, L.E., Cavatte, P.C., Detmann, K.C., Sanglard, L., Ronchi, C.P., y Damatta, F.M. (2012). Source strength increases with the increasing precociousness of fruit maturation in field-grown clones of conilon coffee (Coffea canephora). Trees, 26 (4), pp. 1397-1402. 
Rodríguez, N.F., Cavatte, P.C., Silva, P.E., Martins, S.C., Morais, L.E., Medina, E.F., y Damatta, F.M. (2012). Physiological and biochemical abilities of robusta coffee leaves for acclimation to cope with temporal changes in light availability. Physiologia Plantarum, 149 (1), pp. 45-55.
Somarriba, E. (2004). ¿Cómo evaluar y mejorar el dosel de sombra en cacaotales?. Agroforestería en las Américas, 41-42, pp. 120-128.

Taiz, L., y Zeiger, E. (2006). Plant Physiology. $4^{\text {th }}$ ed. Sunderland-Sinauer Associates. Massachusetts, p 764. 
